\title{
Recent Advances in Mercury Research
}

\author{
Ebany J. Martinez-Finley • Michael Aschner
}

Published online: 28 March 2014

(C) Springer International Publishing AG 2014

\begin{abstract}
Mercury (Hg) is a highly toxic, non-essential, naturally occurring metal with a variety of uses. Mercury is not required for any known biological process and its presence in the human body may be detrimental, especially to the nervous system. Both genetic and behavioral studies suggest that mercury levels, age (both of exposure and at testing), and genetic background determine disease processes and outcome. The metal receptors and genes responsible for mercury metabolism also appear to play a pivotal role in the etiology of mercury-induced pathology. This review presents information about the latest advances in mercury research, with particular focus on low-level exposures and the contribution of genetics to toxic outcome. Future studies should address the contribution of genetics and low-level mercury exposure to disease, namely gene $\mathrm{x}$ environment interactions, taking into consideration age of exposure as developing animals are exquisitely more sensitive to this metal. In addition to recent advances in understanding the pathology associated with mercury exposure, the review highlights transport mechanisms, cellular distribution and detoxification of mercury species in the body.
\end{abstract}

Keywords Mercury · Methylmercury · Ethylmercury · Bioaccumulation · Degeneration · Skin creams · Genetics . Autism $\cdot$ pdr-1 $\cdot$ skn-1 $\cdot$ Fish $\cdot$ C. elegans

E. J. Martinez-Finley

The Mind Research Network, Albuquerque, NM, USA

M. Aschner $(\square)$

Department of Molecular Pharmacology, Albert Einstein College of Medicine, Forchheimer 209, 1300 Morris Park Avenue, Bronx, NY 10461, USA

e-mail: michael.aschner@einstein.yu.edu

\section{Introduction}

Mercury (Hg) is a naturally occurring element present in the Earth's crust. Although present in the environment and atmosphere in small quantities, it is a source of concern due to its considerable release through anthropogenic emissions. Mercury toxicity is not limited to humans; it is also detrimental to animals and plants [1]. The mechanisms through which it causes cell death are of particular interest and this has received considerable attention [2•]. Mercury has a rich history; its applications have varied from extracting gold (which remains a common practice in developing countries) to treating everything from constipation to syphilis. Many believe that the character of the 'Mad Hatter' in Alice in Wonderland was based on English hatters who were poisoned by mercuric nitrate used in the felting process [3].

Natural sources of mercury in the environment include elemental mercury vapor from volcanoes and forest fires and the release of inorganic mercury by weathering and movement of water [4]. More recent anthropogenic sources include burning of coal and fossil fuels, mining of mercury, precious metal refinement, electrical and automotive part manufacture, and chemical processing, and release through waste incineration, landfills, and industrial contamination of water systems.

Some of the best-known environmental effects of mercury on human health come from Minamata, Japan, and Iraq secondary to consumption of tainted fish and wheat, respectively. In Minamata, over the course of several years industrial wastewater containing mercury was released into the environment and bioaccumulated in fish. In the 1950s, adults who consumed the mercury-laced fish developed Minamata disease, which was eventually characterized by paresthesia, sensory deficits, slurred speech, unsteady gait, muscle weakness, irritability, memory loss, and depression [2•]. Similar symptoms were observed in Iraq after consumption of bread made from grain treated with ethylmercury [2•]. Methylmercury $(\mathrm{MeHg})$ contamination of 
fish is still a significant source of human exposure to mercury compounds in fish-eating populations.

Because mercury in the environment poses a significant risk to human health it, is regulated by the United States Environmental Protection Agency (US EPA) and the Occupation Health and Safety Agency (OSHA). These agencies take recommendations from the National Academy of Sciences expert panels that review available scientific studies and make safe level recommendations. It is recommended that levels of mercury not exceed $5.0 \mu \mathrm{g} / \mathrm{L}$ in whole blood or $1.0 \mu \mathrm{g} / \mathrm{g}$ hair, which corresponds to a reference dose of $0.1 \mu \mathrm{g} / \mathrm{kg}$ body weight/day [5].

The FDA also limits the amount of mercury in cosmetic products to $1 \mathrm{ppm}$. In a study conducted in 2013, 549 skinlightening products, manufactured in 32 countries, purchased both online and in stores in the USA, Taiwan, Japan, China, Thailand, and Sri Lanka were tested for mercury levels. Cosmetics were screened for mercury content above 200 ppm using a low-cost portable x-ray fluorescence spectrometer. Of the 549 tested products, 33, representing $\sim 6.0 \%$, contained mercury above $1,000 \mathrm{ppm}$. Forty-five percent contained mercury in excess of $10,000 \mathrm{ppm}$ and $3.3 \%$ of skin-lightening products purchased in the USA were found to contain mercury in excess of $1,000 \mathrm{ppm}$ [6].

Mercury is a very potent toxicant, with the nervous system, particularly the developing nervous system, being the most affected. Various mercury research studies have emerged in the last few years and the number is too vast to review all of them here, but common 'themes' have appeared, such as studies of low-level mercury exposure, and the contribution of genetics to mercury toxicity. The most recent of these studies are reviewed herein. A background of mercury administration, distribution, metabolism, and excretion is provided for context.

\section{Mercury Species}

Mercury, also known as quicksilver, is the only metal that is liquid at room temperature. There are three primary forms of mercury: elemental or metallic mercury $\left(\mathrm{Hg}^{0}\right)$, inorganic mercury, and organic mercury. $\mathrm{Hg}^{0}$ is a silver-colored liquid at room temperature but forms mercury vapors at ambient temperatures due to its very high vapor pressure. These characteristics have made mercury particularly valuable for use in production of chlorine gas and caustic soda, extraction of gold and silver from ore, and as a component of batteries, dental amalgam, thermometers, barometers, and electrical switches (Table 1). Elemental mercury is rarely found in nature but can be heat-extracted from inorganic cinnabar ores. Mercuric mercury $\left(\mathrm{Hg}^{2+}\right)$ is the oxidation state that is most commonly found.

$\mathrm{MeHg}$ is the form of mercury that is highly neurotoxic and exerts most of the toxicity commonly associated with mercurials. Depending on the depth and oxygen content of waters, $\mathrm{Hg}^{2+}$ and mercury sulfide $(\mathrm{HgS})$ can be transformed by methanogens and sulfate-reducing bacteria to $\mathrm{MeHg}$. Because MeHg has a higher bioavailability than elemental and inorganic compounds, and because it is highly lipid soluble, it can diffuse out of the cell and can accumulate in waters where it can be absorbed by bottom feeders such as zooplankton and other small organisms. These organisms are consumed by small fish, which are then eaten by larger fish, resulting in mercury bioaccumulation the higher you go up the food chain. Due to bioaccumulation and slow excretion rates, $\mathrm{MeHg}$ is concentrated by as much as a million fold in larger predatory fish relative to the surrounding water [7]; this is particularly problematic for fish that have more fat due to mercury's high lipid solubility [8].

\section{Sources}

Humans absorb $\mathrm{MeHg}$ and $\mathrm{Hg}^{2+}$ naturally through ingestion of contaminated fish and plants. Cellular fractions of muscle tissue from several species of commercially farmed fish were analyzed for mercury species/content. The highest levels of total mercury were found bound to cellular debris and the metallothionein-like pool [9]. This sequestration of mercury at the tissue and cellular level may reduce the toxicity of mercury to the fish, but unfortunately contributes to the high concentrations of mercury present in fish.

Occupational exposure and anthropogenic mercury emissions can also present significant sources of mercury exposure to humans. Human exposure to $\mathrm{Hg}^{0}$ can occur through dermal contact, ingestion, and/or through inhalation of vapors. Occupations with reported mercury exposures are waste disposal, electrical and auto parts manufacturing, chemical processing, metal processing, dental occupations, construction, and house painting. Non-dietary, non-occupational sources of exposure are mercury spills from broken light bulbs or thermometers and from dental fillings.

\section{Absorption, Transport, and Detoxification}

The absorption, transport, and detoxification of mercury is dependent on the mercury species. For elemental and inorganic mercury, inhalation of mercury vapors is the most significant source of absorption, with as much as $80 \%$ of the inhaled vapors directly entering the bloodstream. Upon absorption, elemental mercury enters all tissues and accumulates in the central nervous system and kidneys. Inorganic mercury has low lipid solubility and does not readily cross cell membranes. Once absorbed, the majority of $\mathrm{Hg}^{2+}$ accumulates in the kidney and liver (Table 1).

As much as $90 \%$ of ingested $\mathrm{MeHg}$ is absorbed through the intestine and forms thiol (-SH) complexes with proteins 


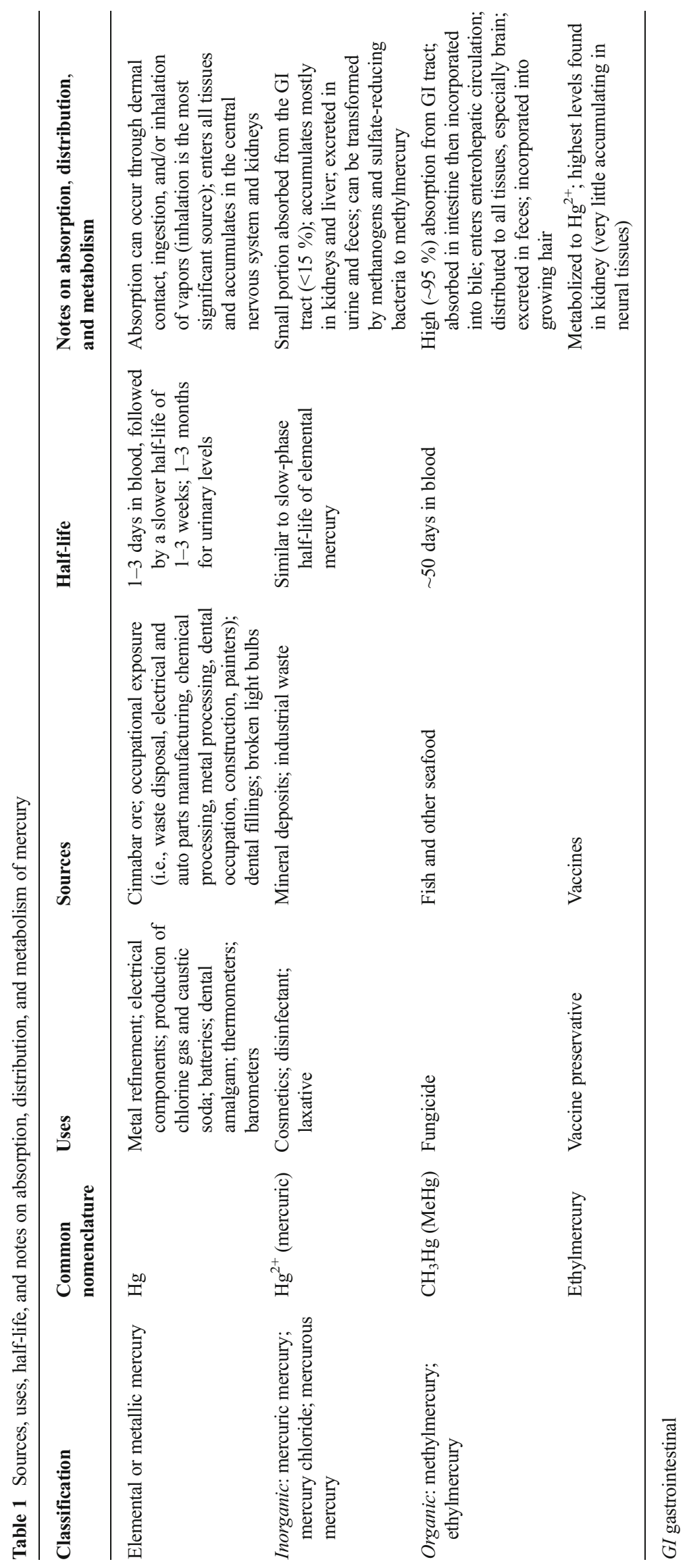


and amino acids [such as glutathione (GSH) and cysteine] in the liver. Some MeHg enters the general circulation, where it is distributed throughout all tissues including the brain (Table 1). However, most of the absorbed MeHg is incorporated into bile, secreted into the intestine, and reabsorbed through the enterohepatic circulation. Other organomercurials, such as ethylmercury, are metabolized to $\mathrm{Hg}^{2+}$ more rapidly (the longer the alkyl chain of the organic mercury compound, the more it behaves as inorganic $\mathrm{Hg}^{2+}$ ), and therefore have different tissue accumulation. Similar to $\mathrm{Hg}^{2+}$, the highest levels of ethylmercury are found in the kidney, with very little accumulating in neural tissues $[10 \bullet, 11]$.

Mercury has high affinity for thiol (-SH) and selenol (-SeH) groups; these groups are used primarily for mercury transport both through cells and through the body. Metallothionein and GSH are examples of thiol-rich intracellular proteins. Selenoproteins include GSH peroxidase, iodothyronine deiodinase, thioredoxin reductase, and selenoprotein $\mathrm{P}$. Cysteine residues on serum albumin and hemoglobin are used to transport $\sim 90 \%$ of $\mathrm{Hg}^{2+}$ and $\mathrm{MeHg}$ in plasma. Globins and other plasma proteins including selenoprotein $\mathrm{P}$ are responsible for the remaining percentage. MeHg in blood is found conjugated with GSH in erythrocytes $[12,13]$.

In addition to transport, detoxification of $\mathrm{Hg}^{2+}$ and $\mathrm{MeHg}$ species can also occur through complexation with GSH. GSH-mercury complexes facilitate systemic excretion through the liver (bile) and kidneys as well as cellular efflux of MeHg from astrocytes [14]. The affinity of inorganic and organic mercury compounds is higher for selenol groups than for thiol groups [15-17].

\section{Neurotoxicity}

It is readily established that $\mathrm{MeHg}$ disrupts the nervous system, yet the mechanism of damage remains poorly understood. Before mercury can have an effect, it must first cross several barriers. Several studies support that MeHg-L-cysteine is transported across the blood-brain barrier by the L-type amino acid transporters used to transport neutral amino acids [18], as was first observed by Aschner et al., who observed competitive inhibition of $\mathrm{MeHg}$ uptake in astrocytes by large, neutral amino acids $[19,20]$.

\section{Low-level Mercury Exposure Studies (Humans)}

Few studies have evaluated the neurobehavioral effects of mercury exposure in adult populations. A Korean group of researchers [21] studied the association between low-level mercury exposure and neurobehavioral functions in adults aged 20-65 years living in coastal regions of the country. The mean mercury level in the blood was $5.41 \mu \mathrm{g} / \mathrm{L}$, in urine was $1.17 \mu \mathrm{g} / \mathrm{g}$-creatinine, and in hair was $1.37 \mathrm{mg} / \mathrm{kg}$. The only significant result was an association between right-hand finger taps and urine mercury levels, which suggests that lowlevel mercury exposure in adults is not associated with deficits in neurobehavioral functions [21].

The objective of the Flemish biomonitoring program FLEHS I, conducted from 2002 to 2006, was to study regional exposure levels in an attempt to link possible differences in internal exposure levels to local environmental pressure. In the second installment of the program, FLEHS II, conducted from 2007 to 2011, the main objectives were to determine reference levels of mercury in hair for the population of Flanders, Belgium, to assess relationships between mercury exposure and possible sources such as fish consumption, and to assess dose-effect relationships between mercury exposure and health effect markers. The study consisted of approximately 200 adolescents and 250 mothers as a reference. The results revealed that mercury concentrations in the Flemish population were rather low compared with other studies. Mercury levels in the Flemish populations were strongly related to the age of the participants and seafood intake. Asthma, having been breastfed as a newborn, age of menarche, allergy for animals, and free testosterone levels were negatively associated with mercury in hair. Janus kinase 2 (JAK2), ARID4A, Hist1HA4L (boys) and HLAdrb5, PIAS2, MANN1B1, GIT and ABCA1 (girls) were also significantly correlated with mercury levels in hair [22].

In addition to Minamata and Iraq, there was another mercury epidemic in Niigata, Japan, in the 1960s. During this epidemic two extensive surveys were collected, but cases diagnosed after the epidemic or those exposed to low levels of $\mathrm{MeHg}$ (hair mercury level $<50 \mu \mathrm{g} / \mathrm{g}$ ) were not welldocumented. From these two surveys, Maruyama and colleagues examined 103 adults with hair mercury measurements and compared the prevalence and distribution of neurological signs related to $\mathrm{MeHg}$ poisoning between exposure categories. They found 48 subjects with neurological signs, such as sensory disturbance of bilateral distal extremities, disequilibrium, hearing impairment and ataxia, in the $\mathrm{MeHg}$ group with a hair mercury concentration $<50 \mu \mathrm{g} / \mathrm{g}$ [23].

A study conducted in Hong Kong children examined the association between low-dose prenatal $\mathrm{MeHg}$ exposure and neurocognitive outcomes. 608 subjects were assessed using the Hong Kong Wechsler Intelligence Scale for Children (HK-WISC), Hong Kong List Learning Test (HKLLT), Tests of Everyday Attention for Children (TEACH), Boston Naming Test, and Grooved Pegboard Test. After correcting for child age and sex, multivariate analysis showed a negative correlation between the cord blood mercury concentration and the Picture Arrangement of HK-WISC, and Short and Long Delay Recall Difference of the HKLLT subtests. Small adverse associations were also found between prenatal $\mathrm{MeHg}$ exposure and longterm neurocognitive effects as measured via the visual sequencing task and retention ability of verbal memory [24]. 


\section{Low-level Mercury Exposure Studies (Animals and Cell Models)}

A number of studies have evaluated the role of low-dose mercury in neurodevelopmental toxicity in animal models. The effects of low-dose prenatal MeHg on neuronal migration in the developing rat cerebral cortex were assessed using in utero electroporation. Offspring of rats exposed prenatally during the critical stage in neuronal migration exhibited migration defects of the cerebrocortical neurons. Altered proliferation of neural progenitor cells (NPCs), induction of apoptosis of NPCs or newborn neurons, abnormal differentiation of NPCs, and the morphological changes of radial glial scaffold were ruled out as the sources of the abnormal neuronal distribution, suggesting that the defective neuronal positioning triggered by exposure to a low-dose of $\mathrm{MeHg}$ was due to the impacts of $\mathrm{MeHg}$ on the process of neuronal migration itself. In utero exposure to lowlevel MeHg also suppressed the expression of Rac1, Cdc42, and RhoA, key players in migration of cerebrocortical neurons during the early stage of brain development [25].

To examine whether low doses of $\mathrm{MeHg}(30-300 \mathrm{nM})$ and mercury chloride $\left(\mathrm{HgCl}_{2}\right)$ could modulate the activity of the JAK/signal transducer and activator of transcription (STAT) signaling pathway, Jebbett and colleagues [26] used human SH-SY5Y neuroblastoma and mouse cortical NPCs. They showed enhanced ciliary neurotrophic factor (CNTF)-evoked STAT3 phosphorylation in both cell lines following subcytotoxic doses of MeHg. This effect was specific for $\mathrm{MeHg}$, as $\mathrm{HgCl}_{2}$ did not enhance signaling. The NPCs further showed enhanced CNTF-induced expression of STAT3 target genes such as glial fibrillary acidic protein (GFAP) and suppressed cytokine signaling 3 (SOCS3), and increased the proportion of cells expressing GFAP following 2 days of differentiation following $\mathrm{MeHg}$ exposure. Higher, nearcytotoxic concentrations of $\mathrm{MeHg}$ and $\mathrm{HgCl}_{2}$ inhibited STAT3 phosphorylation and led to increased production of superoxide. Lower concentrations of MeHg activated JAK/ STAT signaling but did not result in a detectable increase in superoxide or increased expression of heme oxygenase 1, heat shock protein A5, and sirtuin 1. These findings suggest that low concentrations of MeHg can enhance STAT3 phosphorylation and glial differentiation through an alternative mechanism than that of reactive oxygen species (ROS) and cell death [26].

\section{Contribution of Genetics to Methylmercury Toxicity}

Recent studies suggest that several genes mediating the toxicokinetics of mercury are polymorphic in humans and may be responsible for inter-individual variability.

Using a subsample $(n=1,311)$ of the Avon Longitudinal Study of Parents and Children (Bristol, UK), Grandjean and colleagues [27] measured mercury content from freeze-dried umbilical cord tissue as well as sociodemographic cofactors, nutrition, genetic polymorphisms, and the Wechsler Intelligence Scale for Children Intelligence Quotient (IQ) scores at age 8 years to account for cognitive ability [28]. Children in this study had low background mercury exposures. They showed a positive association between $\log 10$ transformed mercury and IQ scores, but this association did not withstand adjustment for nutritional and sociodemographic co-factors. The authors used the Illumina ${ }^{\circledR}$ HumanHap550 quad chip genotyping platform by 23andMe to look for single nucleotide polymorphisms (SNPs) on candidate genes belonging to brain development and neurotransmitter metabolism, cholesterol metabolism, iron regulation, and peroxidative defense. $\mathrm{MeHg}$ interactions ( $\mathrm{MeHg} \times$ total IQ) were detected for three SNPs including paraoxonase 1 (rs662), progesterone receptor (rs 1042838), and brainderived neurotrophic factor (rs2049046), and transferrin (rs3811647) was associated with mercury concentrations. There were only equivocal associations between $\mathrm{MeHg}$ exposure and adverse neuropsychological outcomes. Interestingly, they did find heterogeneities in several relevant genes, which the authors suggest could mean an increased predisposition to $\mathrm{MeHg}$ neurotoxicity [27].

In a study in dental professionals, Goodrich and colleagues studied polymorphisms in key GSH-synthesizing enzyme, GSH s-transferase, and selenoprotein genes underlying interindividual differences in mercury body burden. They used urine as a biomarker for elemental mercury and hair as a biomarker for $\mathrm{MeHg}$ and concluded that the average urine $(1.06 \pm 1.24 \mu \mathrm{g} / \mathrm{L})$ and hair mercury levels $(0.49 \pm 0.63 \mu \mathrm{g} / \mathrm{g})$ were similar to national US population averages. TaqMan assays were used to genotype DNA from buccal swab samples at 15 polymorphic sites in genes implicated in mercury metabolism. Linear regression modeling assessed the ability of polymorphisms to modify the relationship between mercury biomarker levels and exposure sources (e.g., amalgams, fish consumption). Five polymorphisms were significantly associated with urine mercury levels (GSTT1 deletion), hair mercury levels (GSTP1-105, GSTP1-114, GSS 5'), or both (SEPP1 3'UTR). This study suggests that polymorphisms in selenoproteins and GSH-related genes may influence elimination of elemental mercury and $\mathrm{MeHg}$ [28].

Autism is a common neurodevelopmental disorder with suspected genetic and environmental components. Genetic susceptibility to high mercury has been suggested as an autism risk factor for some children. Owens and colleagues [29] hypothesized that mercury could be implicated in the etiology of autism if genetic susceptibility altered its metabolism or intracellular compartmentalization. Genetic sequences of four genes, L-type amino acid transporter 1 (LAT1), divalent metal transporter 1 (DMT1), metal-regulatory transcription factor 1 (MTF1), and metallothionein 1a (MT1a), implicated in the 
transport of and response to mercury were screened for variation and association with autism. They identified and characterized 74 variants in these four genes and polymorphisms through screening 48 unrelated individuals from the general and autistic populations who were evaluated for differences in allele frequencies. Analysis failed to show an association with autism for any variant evaluated, suggesting that variations in the ability of these genes to process and transport mercury may not play a significant role in the etiology of autism [29].

Another study examined the role of the endothelial nitric oxide (eNOS) gene polymorphisms, T-786C and Glu298Asp, on nitrite concentrations following mercury exposure in humans under the premise that mercury exposure might decrease circulating nitrite concentrations and that variants in the eNOS gene might enhance the adverse effects of mercury, resulting in increased risk of cardiovascular disease. Blood samples were collected from volunteers exposed to $\mathrm{MeHg}$ following fish consumption. There were no significant differences in age, arterial blood pressure, body mass index, heart rate, and concentrations of mercury and nitrite concentrations between the genotype groups. Decreased nitric oxide (NO) production was attributed to mercury, age, and gender and was not influenced by gene polymorphisms. Their findings suggest that T-786C and Glu298Asp were not associated with an increased risk for cardiovascular diseases in MeHg-exposed subjects [30]. The same group investigated the contribution of the 27-nt tandem repeat of intron 4 of the eNOS gene on NO production, which could enhance susceptibility to cardiovascular disease in the MeHg-exposed study population. They found no significant differences in age, arterial blood pressure, body mass index, or cardiac frequency between genotype groups but observed lower nitrite levels for the 4a4a genotype carriers. Age, gender, and the presence of the intron 4 polymorphism contributed to nitrite reduction as a result of the blood mercury concentration. Their results suggested that the $27 \mathrm{nt}$ repeat polymorphism of intron 4 in the eNOS gene increases susceptibility to cardiovascular diseases after $\mathrm{MeHg}$ exposure by modulating NO levels [31].

The n-3 polyunsaturated fatty acids eicosapentaenoic acid and docosahexaenoic acid, which are present in fish, are protective against myocardial infarction (MI). However, the risk of MI can also be influenced by $\mathrm{MeHg}$ contamination of fish. Engström and colleagues elucidated whether genetic polymorphisms in GSH-related genes modify the association between eicosapentaenoic + docosahexaenoic acid or $\mathrm{MeHg}$ and the risk of first-ever MI. Individuals from northern Sweden were genotyped to examine polymorphisms in GSH-synthesizing or GSH-conjugating genes. The impact of these polymorphisms on the association between erythrocytemercury (proxy for $\mathrm{MeHg}$ ) and the risk of MI, as well as between plasma eicosapentaenoic + docosahexaenoic acid and the risk of MI, was evaluated, while also considering the effect of erythrocyte-selenium on the risk of MI. There were no strong genetic modifying effects between plasma eicosapentaenoic + docosahexaenoic acid or erythrocyte-Hg and risk of MI. No statistically significant genetic modifying effects were seen for the association between plasma eicosapentaenoic + docosahexaenoic acid or erythrocytemercury and the risk of MI (summarized in Table 2) [32].

Resistance to mercury is widely distributed among bacteria isolated from healthy adults and children, with the presence of mercury resistance genes almost always associated with the concomitant presence of antibiotic resistance genes [33-40]. This association between antibiotic resistance and heavy metal resistance has also been described in Staphylococcus aureus.

Studies in Caenorhabditis elegans conducted by MartinezFinley and colleagues have shown a latency period for neurodegeneration in combination with susceptibility of genetic background $[41,42]$. The first study described the impact of early-life exposure to $\mathrm{MeHg}$ and knockdown of $s k n-1$, the major stress-activated cytoprotective transcription factor, on dopaminergic (DAergic) neurodegeneration, mercury content, and stress reactivity in the nematode $C$. elegans. Mercury content was not strain dependent; however, the generation of ROS was dramatically increased in $s k n-1$ knockout (KO) compared with wild-type worms. Structural integrity of DAergic neurons was microscopically assessed by visualization of fluorescently-labeled neurons, and revealed loss of neurons in skn-1KO- and MeHg-exposed worms compared with wild-type controls. Dopamine-related behavior in the worms was decreased in response to $\mathrm{MeHg}$ exposure and was decreased in $s k n-1 \mathrm{KO}$ worms; both were apparent only after a period of latency. Combined, these studies suggest that knockdown of $s k n-1$ in the nematode increases DAergic sensitivity to $\mathrm{MeHg}$ exposure following a period of latency [41].

The second study examined the impact of early-life exposure to $\mathrm{MeHg}$ and $\mathrm{KO}$ of $p d r-1$ (mammalian: parkin/PARK2) on measures of stress reactivity, mercury content, and DAergic degeneration under the premise that KO of $p d r-1$ would exacerbate $\mathrm{MeHg}$ toxicity and damage to the DAergic system. $p d r-1 \mathrm{KO}$ worms showed increased lethality and decreased lifespan following $\mathrm{MeHg}$ exposure and also had increased mercury content relative to wild-type worms. ROS were significantly increased in both strains following $\mathrm{MeHg}$ exposure; however, while N2 worms showed an increase in $s k n-1$ transcript levels following $\mathrm{MeHg}$, there was no difference in skn-1 induction in $p d r-1 \mathrm{KO}$ worms, suggesting that they were unable to mount a proper stress response. Dopamine-dependent behavioral analysis revealed an effect of $\mathrm{MeHg}$ on N2 wild-type worms, but no effect on $p d r-1 \mathrm{KO}$ worms. These results suggested that $p d r-1 \mathrm{KO}$ worms were more sensitive to $\mathrm{MeHg}$ than wild-type worms, but $\mathrm{MeHg}$ did not exacerbate behavioral changes already present in pdr$1 \mathrm{KOs}$, even after a period of latency [42]. 
Table 2 Summary of genetic studies in humans

\begin{tabular}{|c|c|c|c|}
\hline Population & Measured & Significant findings & Reference \\
\hline $\begin{array}{l}\text { Subset of the Avon } \\
\text { Longitudinal Study } \\
\text { of Parents and Children } \\
\text { (Bristol, UK) }\end{array}$ & $\begin{array}{l}\text { Hg content from freeze-dried umbilical } \\
\text { cord tissue; sociodemographic cofactors, } \\
\text { nutrition, genetic polymorphisms; } \\
\text { Wechsler Intelligence Scale for } \\
\text { children IQ at age } 8 \text { years }\end{array}$ & $\begin{array}{l}\mathrm{MeHg} \times \text { total IQ interactions detected for } \\
\text { paraoxonase } 1 \text { (rs662), progesterone } \\
\text { receptor (rs1042838), and brain-derived } \\
\text { neurotrophic factor (rs2049046); } \\
\text { transferrin (rs3811647) were } \\
\text { associated with } \mathrm{Hg} \text { concentrations }\end{array}$ & Julvez et al., 2013 [27] \\
\hline Dental professionals & $\begin{array}{l}\text { Polymorphisms in key GSH-synthesizing } \\
\text { enzymes }\end{array}$ & $\begin{array}{l}\text { Five polymorphisms were significantly } \\
\text { associated with urine Hg levels } \\
\text { (GSTT1 deletion), hair Hg levels } \\
\text { (GSTP1-105, GSTP1-114, GSS 5'), } \\
\text { or both (SEPP1 3'UTR) }\end{array}$ & Goodrich et al., 2011 [28] \\
\hline $\begin{array}{l}\text { General and autistic } \\
\text { populations }\end{array}$ & $\begin{array}{l}\text { Genetic sequences of four genes, L-type } \\
\text { amino acid transporter } 1 \text { (LAT1), } \\
\text { divalent metal transporter } 1 \text { (DMT1), } \\
\text { metal-regulatory transcription factor } \\
\text { 1 (MTF1), and metallothionein } \\
\text { 1a (MT1a) }\end{array}$ & $\begin{array}{l}\text { Analysis failed to show association } \\
\text { with autism for any variant evaluated, } \\
\text { suggesting that variations in the ability } \\
\text { of these genes to process and transport } \\
\mathrm{Hg} \text { may not play a significant role in } \\
\text { the etiology of autism }\end{array}$ & Owens et al., 2011 [29] \\
\hline $\begin{array}{l}\text { Volunteers exposed } \\
\text { to MeHg following } \\
\text { fish consumption }\end{array}$ & $\begin{array}{l}\text { Role of eNOS gene polymorphisms } \\
\text { (T-786C and Glu298Asp) on nitrite } \\
\text { concentrations following Hg exposure }\end{array}$ & $\begin{array}{l}\text { - T-786C and Glu298Asp were not } \\
\text { associated with an increased risk for } \\
\text { cardiovascular diseases in MeHg- } \\
\text { exposed subjects } \\
\text { - Age, gender, and the presence of } \\
\text { intron } 4 \text { polymorphism contributed } \\
\text { to nitrite reduction as a result of } \\
\text { blood Hg concentration } \\
\text { - The } 27 \mathrm{nt} \text { repeat polymorphism of } \\
\text { intron } 4 \text { in the eNOS gene increases } \\
\text { susceptibility to cardiovascular } \\
\text { diseases after MeHg exposure by } \\
\text { modulating NO levels }\end{array}$ & de Marco et al., 2012 [31] \\
\hline $\begin{array}{l}\text { Individuals from } \\
\text { Northern Sweden }\end{array}$ & $\begin{array}{l}\text { Polymorphisms in GSH-synthesizing } \\
\text { or GSH-conjugating genes and } \\
\text { MeHg and risk of MI }\end{array}$ & $\begin{array}{l}\text { No statistically significant genetic } \\
\text { modifying effects were seen for } \\
\text { the association between plasma } \\
\text { eicosapentaenoic }+ \text { docosahexaenoic } \\
\text { acid or erythrocyte-Hg and risk of MI }\end{array}$ & Engström et al., 2011 [32] \\
\hline
\end{tabular}

eNOS endothelial nitric oxide, $G S H$ glutathione, $H g$ mercury, $I Q$ intelligence quotient, $M e H g$ methylmercury, $M I$ myocardial infarction, $N O$ nitric oxide

\section{Conclusion}

Mercury is a highly toxic, non-essential, naturally occurring metal whose mechanism of toxicity has continued to evade researchers. The current literature provides evidence that mercury toxicity is complex; hence, genetic analyses and testing with low-level mercury species is likely to provide novel insight into the mechanism of toxicity. Studies (both genetic and behavioral) suggest that the extent of disease may be modulated not only by mercury levels but also by age (both of exposure and at testing) and genetic background. Several studies have pointed to associations between mercury levels and metal receptors (i.e., transferrin) and proteins important for metabolism of mercury (i.e., GSH-synthesizing enzymes) [27, 28]. However, an analysis in autistic individuals did not reveal an association between autism and mercury transport genes [29].

Low-dose mercury exposure was reported to produce neurological signs, such as sensory disturbance of bilateral distal extremities, disequilibrium, hearing impairment, and ataxia [23]. Low-dose mercury was not, however, associated with deficits in neurobehavioral functions in a study in adults [21], yet a genetic study indicated nominal associations between $\mathrm{MeHg}$ and paraoxonase 1, progesterone receptor, and brain-derived neurotrophic factor and total IQ (at age 8 years) [27].

Two studies examined the contribution of $\mathrm{MeHg}$ to cardiovascular disease and found that polymorphisms in eNOS were not associated with increased risk of cardiovascular disease; however, a polymorphism in intron 4 increased susceptibility to cardiovascular disease after $\mathrm{MeHg}$ exposure by modulating NO levels [30, 32]. In addition, plasma eicosapentaenioic + docosahexaenoic acid or erythrocyte-mercury were not found to be associated with the risk of MI $[30,32]$. Future studies should aim to address the contribution of genetics and lowlevel mercury exposure to disease, taking into consideration the age of exposure and the age of testing. 
Acknowledgments This work was supported by grants from the National Institute of Environmental Health Sciences (NIEHS) R01 ES007331 and ES020852 and by the National Institutes of Environmental Health Loan Repayment Program.

\section{Compliance with Ethics Guidelines}

Conflict of Interest Ebany J. Martinez-Finley and Michael Aschner declare that they have no conflict of interest.

Human and Animal Rights and Informed Consent This article does not contain any studies with human or animal subjects performed by any of the authors.

\section{References}

Papers of particular interest, published recently, have been highlighted as:

\section{- Of importance}

1. Eisler R. Mercury hazards from gold mining to humans, plants, and animals. Rev Environ Contam Toxicol. 2004;181:139-98.

2. Farina M, Rocha JB, Aschner M. Mechanisms of methylmercuryinduced neurotoxicity: evidence from experimental studies. Life Sci. 2011;89(15-16):555-63. This paper provides an important overview of the relationship between calcium homeostasis, alteration of glutamate homeostasis, and oxidative stress in $\mathrm{MeHg}$ toxicity.

3. O'Carroll RE, Masterton G, Dougall N, Ebmeier KP, Goodwin GM. The neuropsychiatric sequelae of mercury poisoning. The Mad Hatter's disease revisited. Br J Psychiatry. 1995;167(1):95-8.

4. Bose-O'Reilly S et al. Mercury exposure and children's health. Curr Probl Pediatr Adolesc Health Care. 2010;40(8):186-215.

5. Agency for Toxic Substances and Disease Registry (ATSDR). Toxicological profile for manganese. Atlanta, GA: U.S. Department of Health and Human Services, Public Health Service. 2000

6. Hamann, C.R., et al., Spectrometric analysis of mercury content in 549 skin-lightening products: Is mercury toxicity a hidden global health hazard? J Am Acad Dermatol, 2013.

7. Ballatori N, Boyer JL. Slow biliary elimination of methyl mercury in the marine elasmobranchs, Raja erinacea and Squalus acanthias. Toxicol Appl Pharmacol. 1986;85(3):407-15.

8. Clarkson TW. The toxicology of mercury. Crit Rev Clin Lab Sci. 1997;34(4):369-403.

9. Onsanit S, Wang WX. Sequestration of total and methyl mercury in different subcellular pools in marine caged fish. J Hazard Mater. 2011;198:113-22.

7. Dorea JG, Farina M, Rocha JB. Toxicity of ethylmercury (and Thimerosal): a comparison with methylmercury. J Appl Toxicol. 2013;33(8):700-11. Outlines some important distinctions between ethylmercury and methylmercury that are important for a thorough understanding of mercury toxicity.

11. Burbacher TM et al. Comparison of blood and brain mercury levels in infant monkeys exposed to methylmercury or vaccines containing thimerosal. Environ Health Perspect. 2005;113(8):1015-21.

12. Clarkson TW, Vyas JB, Ballatori N. Mechanisms of mercury disposition in the body. Am J Ind Med. 2007;50(10):757-64.

13. Rabenstein DL, Isab AA. A proton nuclear magnetic resonance study of the interaction of mercury with intact human erythrocytes. Biochim Biophys Acta. 1982;721(4):374-84.
14. Fujiyama J, Hirayama K, Yasutake A. Mechanism of methylmercury efflux from cultured astrocytes. Biochem Pharmacol. 1994;47(9):1525-30.

15. Dryssen D, Wedborg M. The Sulfur-Mercury(II) System in Natural Waters. Water Air Soil Pollut. 1991;56:507-19.

16. Khan MA, Wang F. Mercury-selenium compounds and their toxicological significance: toward a molecular understanding of the mercury-selenium antagonism. Environ Toxicol Chem. 2009;28(8):1567-77.

17. Sasakura C, Suzuki KT. Biological interaction between transition metals ( $\mathrm{Ag}, \mathrm{Cd}$ and $\mathrm{Hg}$ ), selenide/sulfide and selenoprotein P. J Inorg Biochem. 1998;71(3-4):159-62.

18. Ballatori N. Transport of toxic metals by molecular mimicry. Environ Health Perspect. 2002;110 Suppl 5:689-94.

19. Aschner $\mathrm{M}$ et al. Methylmercury uptake in rat primary astrocyte cultures: the role of the neutral amino acid transport system. Brain Res. 1990;521(1-2):221-8.

20. Aschner M, Eberle NB, Kimelberg HK. Interactions of methylmercury with rat primary astrocyte cultures: methylmercury efflux. Brain Res. 1991;554(1-2):10-4.

21. Kim RB et al. Association between Low-level Mercury Exposure and Neurobehavioral Functions in Korean Adults Living in a Coastal City. Environ Health Toxicol. 2013;28:e2013015.

22. Croes, K., et al., Health effects in the Flemish population in relation to low levels of mercury exposure: From organ to transcriptome level. Int J Hyg Environ Health, 2013.

23. Maruyama $\mathrm{K}$ et al. Methyl mercury exposure at Niigata, Japan: results of neurological examinations of 103 adults. J Biomed Biotechnol. 2012;2012:635075.

24. Lam HS et al. Long term neurocognitive impact of low dose prenatal methylmercury exposure in Hong Kong. Environ Int. 2013;54:59-64

25. Guo BQ et al. Low level prenatal exposure to methylmercury disrupts neuronal migration in the developing rat cerebral cortex. Toxicology. 2013;304:57-68.

26. Jebbett NJ et al. Low level methylmercury enhances CNTF-evoked STAT3 signaling and glial differentiation in cultured cortical progenitor cells. Neurotoxicology. 2013;38:91-100.

27. Julvez J, Smith GD, Golding J, Ring S, Pourcain BS, Gonzalez JR, et al. Prenatal methylmercury exposure and genetic predisposition to cognitive deficit at age 8 years. Epidemiology. 2013;24(5):64350. doi:10.1097/EDE.0b013.

28. Goodrich JM et al. Glutathione enzyme and selenoprotein polymorphisms associate with mercury biomarker levels in Michigan dental professionals. Toxicol Appl Pharmacol. 2011;257(2):301-8.

29. Owens SE et al. Lack of association between autism and four heavy metal regulatory genes. Neurotoxicology. 2011;32(6):769-75.

30. de Marco KC, Braga GU, Barbosa Jr F. Determination of the effects of eNOS gene polymorphisms (T-786C and Glu298Asp) on nitric oxide levels in a methylmercury-exposed population. $\mathrm{J}$ Toxicol Environ Health A. 2011;74(20):1323-33.

31. de Marco KC et al. Intron 4 polymorphism of the endothelial nitric oxide synthase (eNOS) gene is associated with decreased NO production in a mercury-exposed population. Sci Total Environ. 2012;414:708-12.

32. Engström KS et al. Evaluation of the impact of genetic polymorphisms in glutathione-related genes on the association between methylmercury or n-3 polyunsaturated long chain fatty acids and risk of myocardial infarction: a case-control study. Environ Health. 2011;10:33

33. Edlund $\mathrm{C}$ et al. Resistance of the normal human microflora to mercury and antimicrobials after exposure to mercury from dental amalgam fillings. Clin Infect Dis. 1996;22(6):944-50.

34. Liebert CA et al. The impact of mercury released from dental "silver" fillings on antibiotic resistances in the 
primate oral and intestinal bacterial flora. Met Ions Biol Syst. 1997;34:441-60.

35. Pike R et al. Effect of restoration of children's teeth with mercury amalgam on the prevalence of mercury- and antibiotic-resistant oral bacteria. Microb Drug Resist. 2003;9(1):93-7.

36. Pike $\mathrm{R}$ et al. Prevalence and antibiotic resistance profile of mercuryresistant oral bacteria from children with and without mercury amalgam fillings. J Antimicrob Chemother. 2002;49(5):777-83.

37. Ready D et al. The effect of amalgam exposure on mercury- and antibiotic-resistant bacteria. Int J Antimicrob Agents. 2007;30(1):34-9.

38. Ready $\mathrm{D}$ et al. Oral bacteria resistant to mercury and to antibiotics are present in children with no previous exposure to amalgam restorative materials. FEMS Microbiol Lett. 2003;223(1):107-11.
39. Summers AO et al. Mercury released from dental "silver" fillings provokes an increase in mercury- and antibiotic-resistant bacteria in oral and intestinal floras of primates. Antimicrob Agents Chemother. 1993;37(4):825-34.

40. Wireman $\mathrm{J}$ et al. Association of mercury resistance with antibiotic resistance in the gram-negative fecal bacteria of primates. Appl Environ Microbiol. 1997;63(11):4494-503.

41. Martinez-Finley EJ et al. The Role of skn-1 in MethylmercuryInduced Latent Dopaminergic Neurodegeneration. Neurochem Res. 2013;38(12):2650-60.

42. Martinez-Finley EJ et al. Early-life exposure to methylmercury in wildtype and pdr-1/parkin knockout C. elegans. Neurochem Res. 2013;38(8):1543-52. 